\title{
BMJ Open Potential impact of physical distancing on physical and mental health: a rapid narrative umbrella review of meta- analyses on the link between social connection and health
}

\author{
Nexhmedin Morina (D) , ${ }^{1}$ Ahlke Kip, ${ }^{1}$ Thole Hilko Hoppen (D) , ${ }^{1}$ Stefan Priebe, ${ }^{2}$ \\ Thomas Meyer ${ }^{1}$
}

To cite: Morina N, Kip A, Hoppen TH, et al. Potential impact of physical distancing on physical and mental health: a rapid narrative umbrella review of meta-analyses on the link between social connection and health. BMJ Open 2021;11:e042335. doi:10.1136/ bmjopen-2020-042335

- Prepublication history for this paper is available online. To view these files, please visit the journal online (http://dx.doi. org/10.1136/bmjopen-2020042335).

Received 02 July 2020

Revised 31 January 2021

Accepted 19 February 2021

A) Check for updates

(C) Author(s) (or their employer(s)) 2021. Re-use permitted under CC BY-NC. No commercial re-use. See rights and permissions. Published by BMJ.

${ }^{1}$ Institute of Psychology, University of Münster, Münster, Germany

${ }^{2}$ Unit of Social and Community Psychiatry, Queen Mary University of London, Faculty of Medicine and Dentistry, London, UK

Correspondence to Dr Nexhmedin Morina; morina@uni-muenster.de

\section{ABSTRACT}

Background The imperative for physical distancing (mostly referred to as social distancing) during COVID-19 pandemic may deteriorate physical and mental health. We aimed at summarising the strength of evidence in the published literature on the association of physical and mental health with social connection via social isolation, living alone and loneliness.

Methods We conducted a systematic search in April 2020 to identify meta-analyses using the Medline, PsycINF0 and Web of Science databases. The search strategy included terms of social isolation, loneliness, living alone and metaanalysis. Eligible meta-analyses needed to report any sort of association between an indicator of social connection and any physical or mental health outcome. The findings were summarised in a narrative synthesis.

Results Twenty-five meta-analyses met our criteria, of which 10 focused on physical health and 15 on mental health outcomes. The results suggest that lack of social connection is associated with chronic physical symptoms, frailty, coronary heart disease, malnutrition, hospital readmission, reduced vaccine uptake, early mortality, depression, social anxiety, psychosis, cognitive impairment in later life and suicidal ideation.

Conclusions The existing evidence clearly indicates that social connection is associated with a range of poor physical and mental health outcomes. A potential negative impact on these outcomes needs to be considered in future decisions on physical distancing measures.

\section{BACKGROUND}

The COVID-19 pandemic poses a global public health threat. In order to slow the spread of the virus by reducing contact rates, governments around the world have taken unprecedented political decisions that have transformed societies. The exact form and extent of these measures have varied, but they always include some types of physical distancing (mostly referred to as social distancing) making it impossible for people to maintain their normal social life.
Strengths and limitations of this study

- This rapid umbrella review focuses on a timely and societally relevant issue.

- The systematic literature search was conducted in three major databases from inception up to April 2020 warranting an extensive and up-to-date overview on relevant meta-analyses in the field.

- Quality of included meta-analyses was rated with a standardised measure.

- Different indicators of social connection were included.

- The used method did not allow for a quantitative comparison of associations with health outcomes.

In many countries, the restrictions have already been in place for several months. Depending on the further course of the pandemic with potential new waves, restrictions might continue for longer periods of time or be reimposed after periods of loosening or abandoning them. When deciding about imposing, continuing or relaxing measures of physical distancing, governments have to consider and balance different risks. While physical distancing is likely to reduce the risk of spreading the virus, it might generate other risks. These include potential damages to the economy and also possible negative consequences for the health of the population. For a balanced decision on further physical distancing measures, evidence is required on whether the measures are likely to impact a range of health outcomes.

A recent general population survey revealed that physical distancing can increase the lack of social connection. ${ }^{1}$ This may happen when people are prevented from travelling, physical meetings with significant others and in some cases even from leaving their home other 
than for essential activities. Social connection has been suggested as an umbrella term representing the extent to which an individual connects to others. ${ }^{2}$ Three indicators of social connection are commonly used in research: social isolation, living alone and loneliness. ${ }^{3-5}$ Social isolation is a behavioural measure of a person's social network that can-at least in theory-be objectively quantified. Living alone describes a basic characteristic of an individual's social situation that can be associated with reduced social relationships but is not necessarily so. ${ }^{2}$ Loneliness, on the other hand, is an individual's subjective assessment of the quality and quantity of their social relationships, reflecting a belief that they have too few or too poor relationships or both. Accordingly, social isolation and living alone represent structural indicators, whereas loneliness represents a quality measure of social connections. ${ }^{25}$

Although these three indicators capture distinct aspects of social connection, they commonly overlap and are associated with each other. Literature suggests that many individuals are socially isolated or lonely or both and that social isolation and loneliness may occur unequally across age groups. For example, Hawkley and colleagues ${ }^{6}$ reported that loneliness decreased with age through the early 70 s and then increased again. Several studies indicate that at least a fifth of adults report frequent loneliness, ${ }^{78}$ and that more than $40 \%$ of adults aged 60 and older report feeling lonely. ${ }^{9}$

The extent to which individuals are socially isolated can have a profound impact on both physical and psychological well-being. ${ }^{2}$ Social connection is thought to influence health through behavioural and biological pathways. ${ }^{10}$ Several studies demonstrate that social connection is associated with health-relevant behaviours such as lack of physical activity, poorer sleep, obsessive behaviour as well as neuroendocrine dysregulation, ${ }^{10}$ chronic allostatic load, ${ }^{11}$ high blood pressure and poor immune functioning. ${ }^{212} 13$ Furthermore, the magnitude of the effect of social connection on mortality may be equivalent to or exceed the impacts of deleterious behaviours such as excessive drinking or obesity. ${ }^{4}$

Physical distancing may increase lack of social connection and, therefore, have a negative impact on physical and mental health. For weighing up this potential impact in policy decisions, the existing evidence needs to be considered. Against this background, we conducted a systematic umbrella review to synthesise the evidence on the association between social connection and physical and mental health outcomes. As recommended by the WHO, we explored relevant meta-analyses by means of a rapid review of evidence. ${ }^{14}$

\section{METHODS}

To select relevant meta-analyses on the association between social connection and physical or mental health outcomes, we conducted a systematic search on 6 April 2020 using the databases Medline, PsycINFO and Web of Science. We conducted multifield searches (in titles, abstracts and key concepts) using the following terms: social isolation, loneliness, living alone, and metaanaly*, which we combined using the Boolean operators "or" plus "and". The full search string for Medline and PsycINFO was "( (TI Loneliness OR AB loneliness OR SU Loneliness) OR (TI social isolation OR AB social isolation OR SU social isolation) OR (TI living alone OR AB living alone OR SU living alone)) AND (TI meta-analy* OR AB meta-analy* OR SU meta-analy*)".

Relevant outcomes included any sort of physical or mental health outcome. We applied no restrictions on age of participants, applied research designs (ie, crosssectional, longitudinal) or publication language. Furthermore, we did not apply any limits. We first inspected the title and abstract of all hits and then read full texts of the hits that seemed to meet the aforementioned inclusion criteria. The Preferred Reporting Items for Systematic Reviews and Meta-analyses reporting standards were followed to document the process of systematic review selection. ${ }^{15}$

\section{Coding of trial characteristics}

Systematic reviews with a quantitative synthesis of trial results (meta-analysis) were retained. Two reviewers (NM and $\mathrm{THH}$ ) coded and extracted from each meta-analysis several objectively verifiable characteristics: authors and year of publication, inclusion criteria, number of included primary studies, number of participants and their composition by age and health conditions, study design, type of social connection (social isolation/living alone/loneliness) evaluated, clinical outcome, length of follow-up, number of databases searched and search areas. Furthermore, we extracted the main findings on the association between social isolation/living alone/ loneliness and health outcomes (correlation values, ORs or HRs and the corresponding 95\% CIs). With respect to the $95 \%$ CIs, both values greater than one (or both values less than one) represent a significant increase (or decrease) as a function of social connection.

\section{Quality assessment}

The quality of included systematic meta-analyses was independently assessed by two reviewers (AK and TM) using A Measurement Tool to Assess Systematic Reviews-2 (AMSTAR-2) ${ }^{16}$ Following the tool's guidelines, the raters assigned one of four global quality ratings (ie, high, moderate, low or critically low) after consideration of 16 potential critical and noncritical weaknesses. Items addressing the following criteria were considered as critical: clear research question including definitions of population, intervention, control group and outcomes, adequacy of the literature search and adequate assessment and/or consideration of risk of bias in the primary studies. Typically, high and moderate ratings reflect the presence of one or more noncritical weakness, while low and critically low ratings indicate one or more critical weaknesses. Any discrepancies among the independent raters were discussed until consensus was reached. 


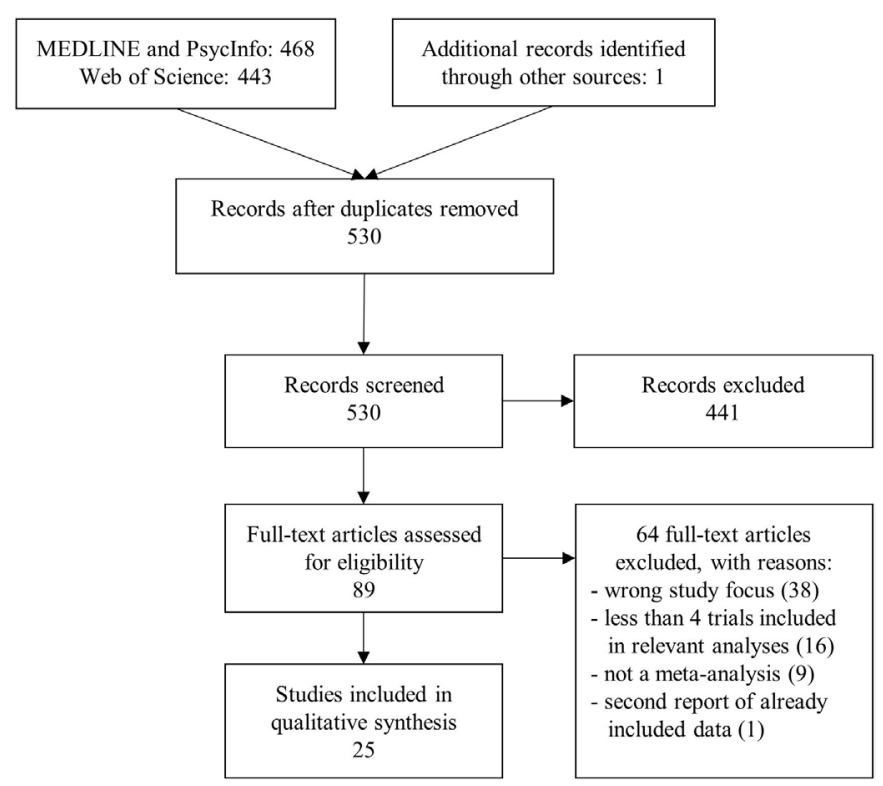

Figure 1 Flow diagram of study selection process.

\section{RESULTS}

\section{Selection and characteristics of included studies}

Figure 1 displays a PRISMA ${ }^{15}$ flow diagram of the publication selection process. After reading 530 abstracts, 89 full-text publications were reviewed. The final review resulted in 25 meta-analyses. Relevant characteristics of these meta-analyses are summarised in table 1 .

All publications were journal articles in English. Ten meta-analyses reported associations of social isolation, living alone and loneliness with physical health outcomes and 15 with mental health outcomes. Different indicators of social connection were measured in the included studies. We considered as structural indicators of social connection: social isolation defined as an objectively quantifiable variable of one's social network ties irrespective of its perceived quality and living alone as an objective characteristic of the living situation. Furthermore, we defined loneliness as a quality indictor representing the subjective emotional appraisal of the extent and quality of social relationships. ${ }^{2}$ The meta-analyses differed with respect to whether they kept these three measures of social connection separate of whether they combined them (see table 1 ).

A total of 276 primary studies were included in the 10 meta-analyses on physical health; however, there was some overlap in samples in meta-analyses that examined cardiovascular disease ${ }^{17} 18$ and early mortality. ${ }^{49}$ Steptoe and Kivimä $\mathrm{ki}^{17}$ and Valtorta $e$ e $a l^{18}$ shared one primary study. In addition, Holt-Lunstad $e t a l^{4}$ and Rico-Uribe et $a l^{19}$ shared 12 primary studies. The reported results in table 1 were based on sample sizes ranging from $1451^{20}$ to $113,374^{21}$ participants, with three meta-analyses not reporting on the sample size. Five meta-analyses were based on longitudinal studies only, one on cross-sectional studies only and the remaining four on a pooled combination of both cross-sectional and longitudinal studies. Furthermore, social isolation and living alone were examined in five meta-analyses on physical health, respectively. Loneliness, on the other hand, was examined in seven meta-analyses on mental health. Only one of these studies was conducted with children and adolescents. ${ }^{22}$ The meta-analyses based on cross-sectional studies revealed a significant association between social connection and the following health problems: chronic physical complaints in children and adolescents, ${ }^{22}$ coronary heart disease and stroke ${ }^{18}$ and frailty in older male (but not female) adults. ${ }^{21}$ Additionally, social connection was associated with malnutrition ${ }^{23}$ and vaccine uptake among older adults. ${ }^{24}$ One meta-analysis ${ }^{20}$ reported mostly nonsignificant results on a positive association between social connection and inflammation (acute-phase $\mathrm{C}$ reactive protein and fibrinogen). The meta-analyses conducted with longitudinal studies indicate that social connection is associated with increased risk of cardiovascular disease, ${ }^{17}$ early mortality ${ }^{49}$ and hospital readmission in patientswith heart failure. ${ }^{25}$

The 15 meta-analyses on mental health were based on a total of 416 primary studies. The reported results are based on sample sizes ranging from $1345^{26}$ to $2,330,163^{27}$ participants, with one meta-analysis failing to report on the sample size. There was some overlap in samples in the four meta-analyses focusing on cognitive functioning or risk of dementia. ${ }^{27-30}$ Kuiper $e t a l^{29}$ shared two primary studies with Evans et $a l,{ }^{28}$ four with Lara $e t a l^{00}$ and three with Penninkilampi et al. ${ }^{27}$ Penninkilampi $e t a l^{27}$ and Lara et $a l^{30}$ further shared two primary studies. Four of the 15 meta-analyses provided longitudinal data only, one provided cross-sectional data only and the remaining 10 meta-analyses reported on both cross-sectional and longitudinal studies. In addition, social isolation, living alone and loneliness were examined in 5, 5 and 10 meta-analyses on mental health, respectively. Three meta-analyses focused on studies with children and adolescents. ${ }^{31-33}$ The included meta-analyses based on cross-sectional designs reported a significant positive association between social connection and late-life suicidal ideation, ${ }^{34}$ depression in adults, ${ }^{35}$ late life depression, ${ }^{26}$ psychosis, ${ }^{31}{ }^{36}{ }^{37}$ smoking behaviour in adolescents, ${ }^{32}$ depression and social anxiety in childhood and adolescence ${ }^{3133}$ and social anxiety disorder in adults. ${ }^{38}$ The meta-analyses based on longitudinal studies suggest that social connection is associated with higher risk of depression in adults, ${ }^{39}$ postacute coronary syndrome depression ${ }^{40}$ and dementia and cognitive impairment in later life. ${ }^{27-30}$ See table 1 for detailed information.

\section{Study quality}

The intraclass correlation coefficient of the global quality ratings among the two raters was $0.83,95 \%$ CI 0.62 to 0.93 , indicating good inter-rater reliability. Study quality was very heterogeneous among meta-analyses both on physical and mental health (see table 1). With respect to the meta-analyses on physical health, the global rating was high in $40 \%$, medium in $10 \%$, low in $40 \%$, critically low in $10 \%$ of the meta-analyses. In the 15 meta-analyses on mental health, study quality was rated as high in $13 \%$, 


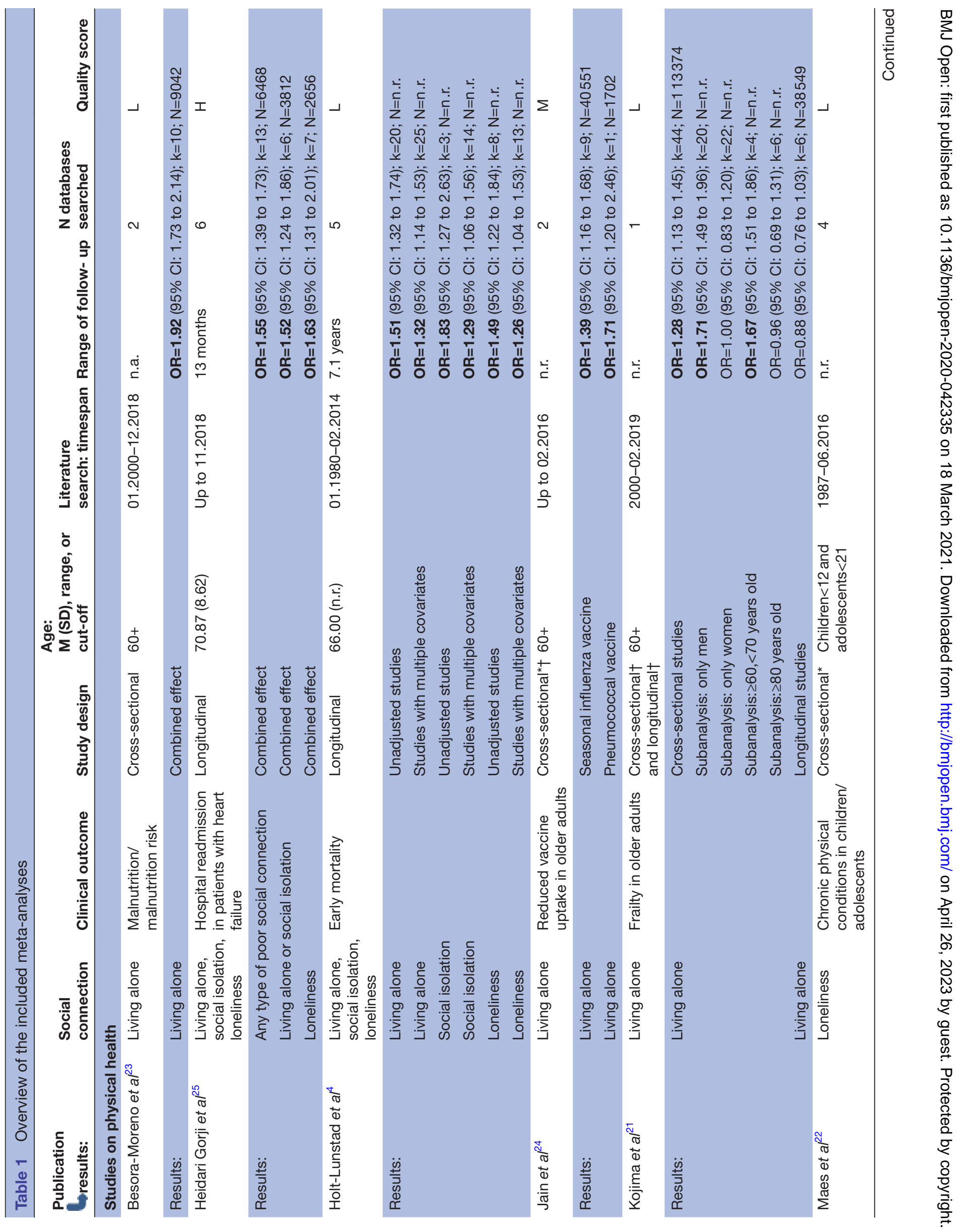




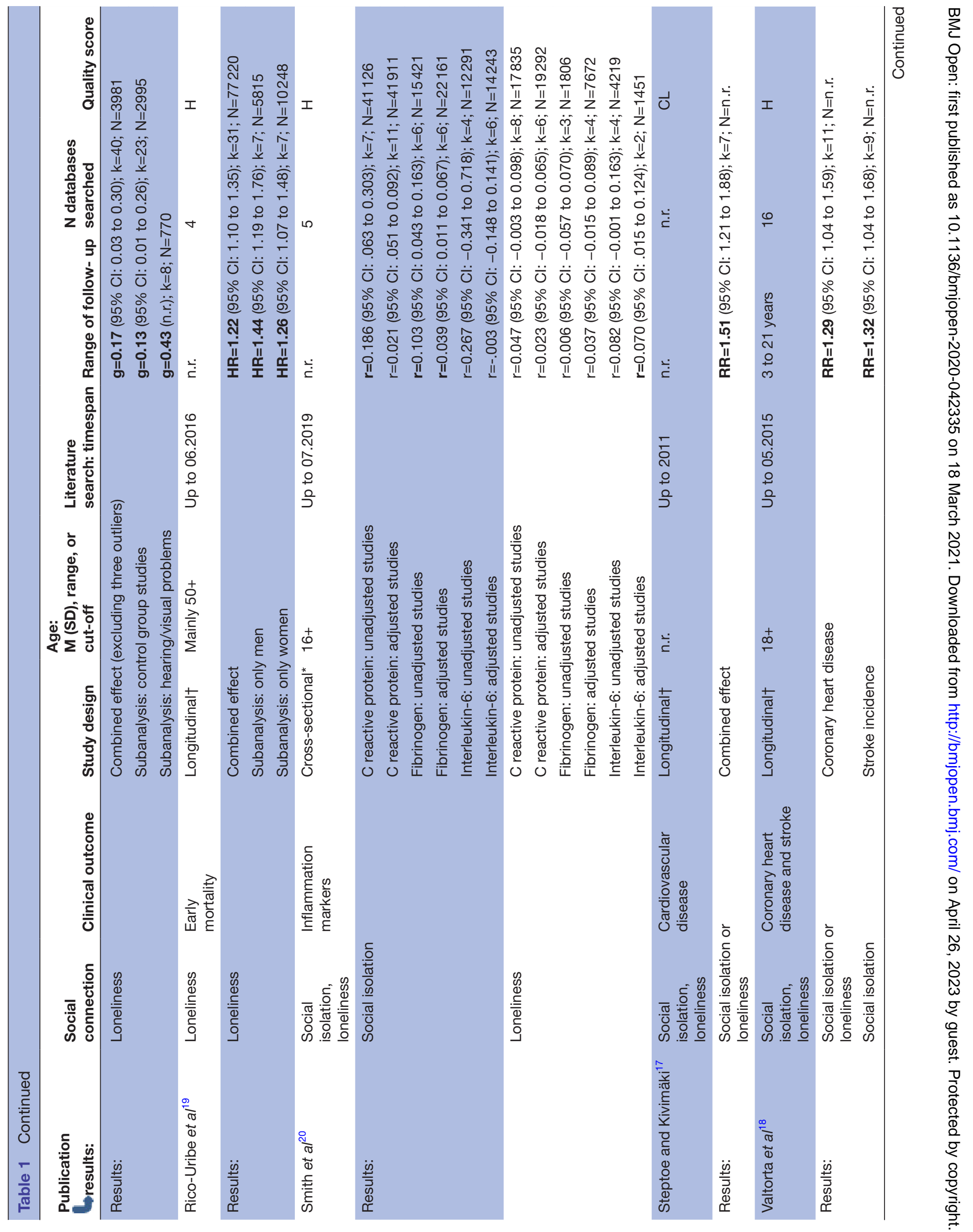




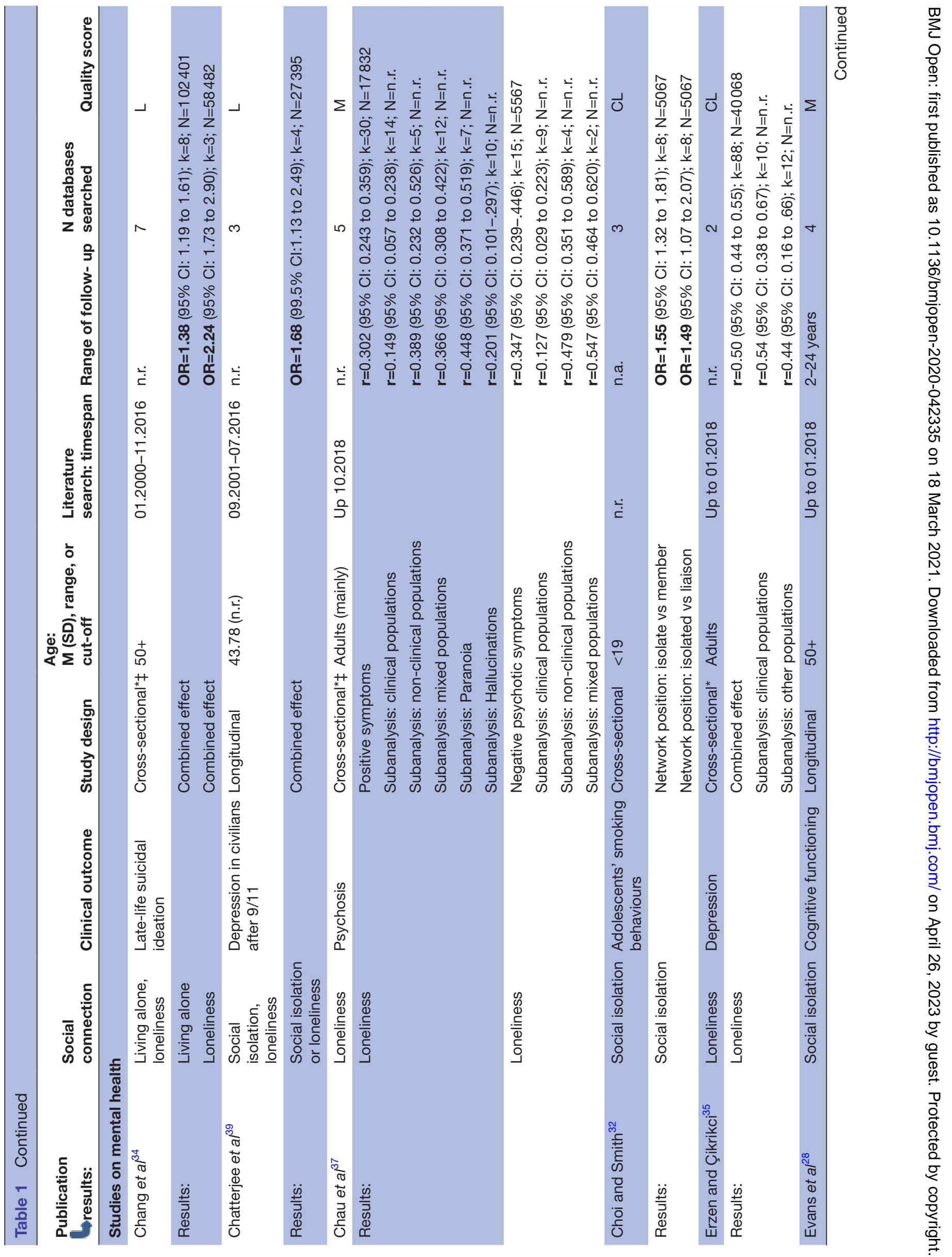




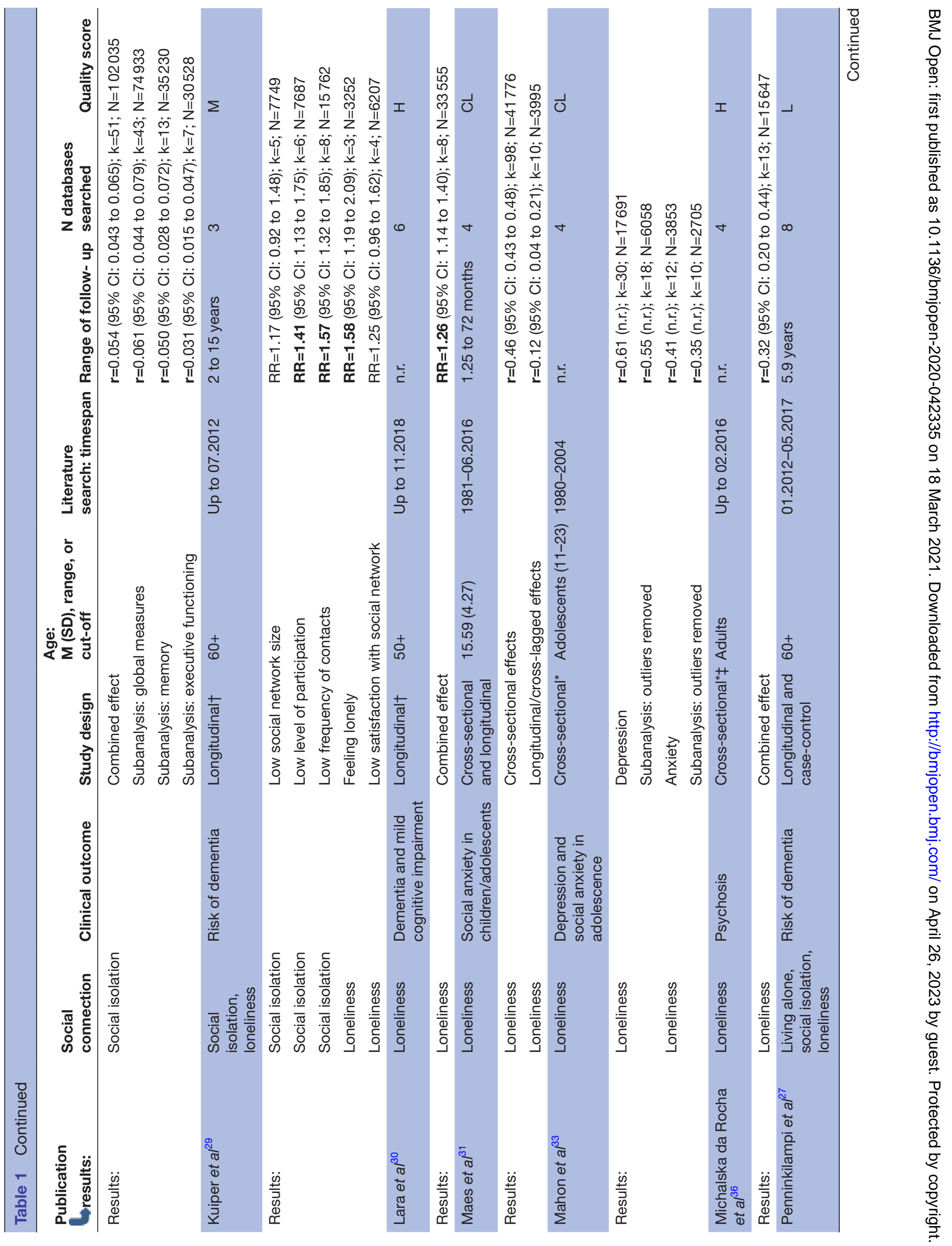




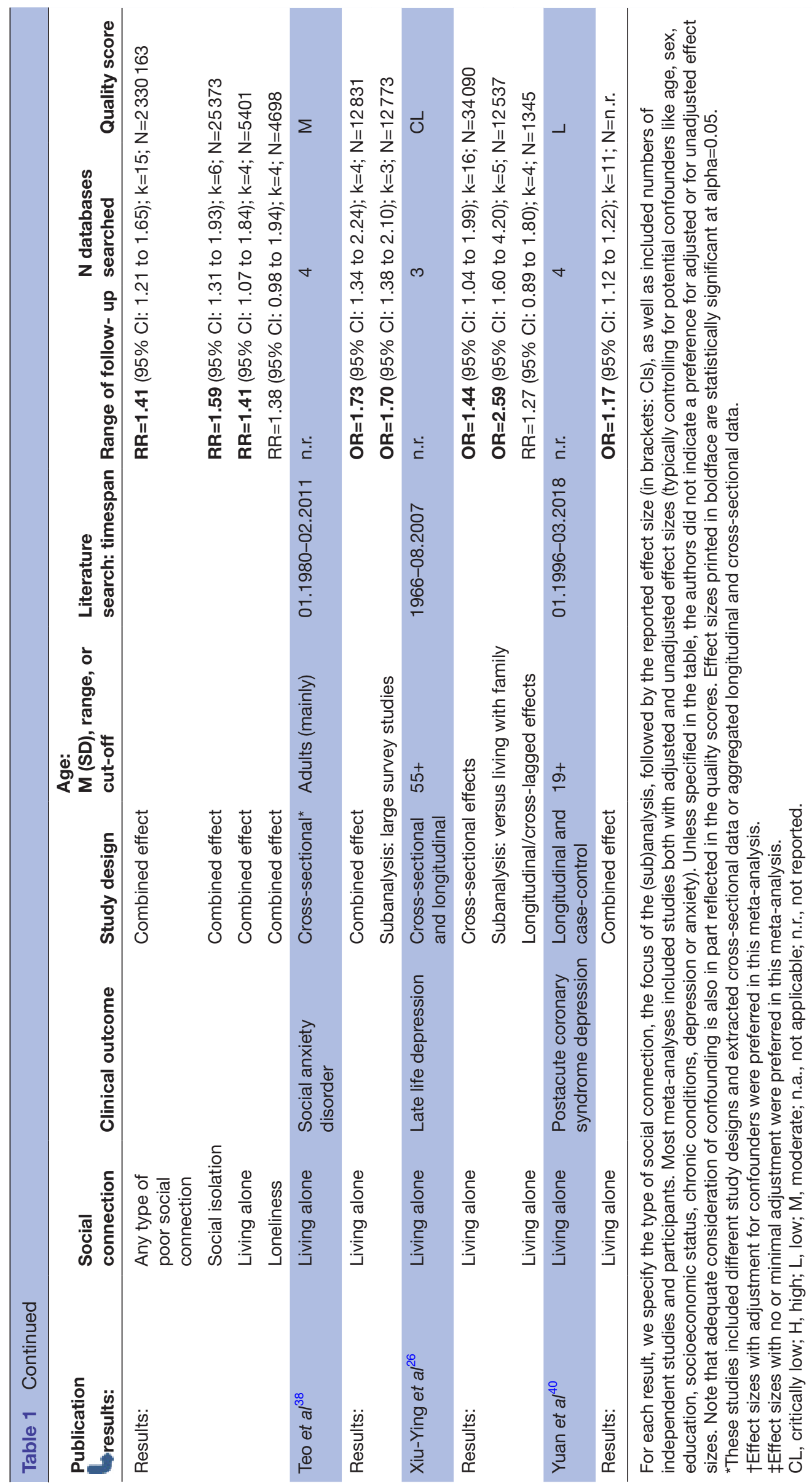

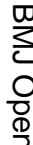


medium in $27 \%$, low in $27 \%$ and critically low in $33 \%$ of the meta-analyses. Among the AMSTAR-2 criteria, inadequate assessment of risk of bias and/or lack of consideration of risk of bias represented the most frequent critical weaknesses of included meta-analyses.

\section{DISCUSSION}

The review clearly demonstrates that lack of social connection is associated with poorer health. This applies to a range of physical and mental health outcomes and has been found in different populations and contexts. The evidence based on both cross-sectional and longitudinal data is substantial for physical health outcome and even more extensive for mental health outcomes. More specifically, social connection is linked with chronic physical symptoms, frailty, coronary heart disease, stroke, early mortality, malnutrition, hospital readmission in patientswith heart failure and vaccine uptake. With respect to mental health, social connection is linked with depression in young and adult populations, social anxiety, psychosis, dementia and cognitive impairment in later life and late-life suicidal ideation.

\section{Strengths and limitations}

This is, to our knowledge, the first review to synthesise the existing evidence that has been reported in meta-analyses on the link between social connection and physical and mental health outcomes. The findings reflect a reasonable number of meta-analyses. Thus, the overall conclusions of this umbrella review are based on an extensive body of empirical evidence.

However, the review also has several limitations. First, we considered different indicators of social connection, and our method did not allow us to identify whether one indicator is more relevant than another. Second, half of the included meta-analyses for both physical and mental health outcomes had an overall quality rated on AMSTAR-2 as low or critically low, with inadequate consideration of risk of bias being the most frequent critical flaw. Third, the quality of the primary research studies that went into the included meta-analyses also varied and their different methodological shortcomings cannot be adequately considered in this review. Fourth, the results on the association between living alone and health outcomes need to be interpreted with caution. As reported above, living alone is not necessarily indicative of feeling lonely. ${ }^{3}$ Finally, the review included a wide range of health outcomes and did not quantify the strength of the associations for different outcomes.

\section{Implications}

The review leaves little doubt that social connection is linked with poorer physical and mental health. The findings are strengthened by the fact that several meta-analyses were conducted with longitudinal studies. In particular, longitudinal data indicate that social connection is associated with increased risk of several physical and mental health outcomes, cardiovascular disease, hospital readmission in patientswith heart failure, early mortality, cogni-

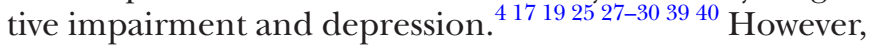
the findings are all based on observational studies and do not provide evidence on the causal direction of the association. Poor physical and mental health can lead to lack of social connection, and lack of social connection can lead to poorer health. For establishing a causal relationship and examining the strength of the predictive relationship of social isolation and loneliness with health outcomes experimental studies are required, which were not the subjects of this review. ${ }^{51}$ Experimental research with animals, however, suggests that lack of social connection increases mortality. ${ }^{42}$ Furthermore, experimental studies with humans indicate that randomly inducing loneliness or exclusion leads to different health-relevant physiological responses than being randomly assigned to a support condition. ${ }^{42}$ For most of the considered outcomes in this review, a causal effect of social connection is plausible and likely to explain at least part of the identified associations. The casual direction is definite in case of the greater risk of isolated people to die early. ${ }^{4}$ For an explanation of the damaging effect of social connection on health outcomes, one may refer to different theoretical models. Theorists from different perspectives have postulated that the impact of social connection on health is mediated by impairments in social capital, ${ }^{43}$ social control, ${ }^{44}$ social identification $^{45}$ and social support. ${ }^{46}$

Furthermore, some evidence from randomised controlled trials, however, suggests that expanding the social connections of individuals, for example, through befriending programmes, may indeed improve different health outcomes. ${ }^{47}$ Altogether, the literature on interventions to reduce loneliness and social isolation indicates that a policy focus on social connection is a cost-effective strategy for enhancing health at the population level due to the potential pay-offs in healthcare costs that would otherwise occur. Existing volunteer friendly visiting programmes or psychosocial group interventions ${ }^{48}$ may need to be redesigned to the point that they can be readily implemented in accordance with existing rules of physical distancing. Creative programmes and interventions to foster social connections, including technology-based social networking programmes, are needed. ${ }^{49}$ Furthermore, existing policies should ensure that populations at greater risk, such as the poor, receive most support. ${ }^{1}$

All the included studies assessed social connection as it occurs in a normal societal context. Physical distancing as part of measures to limit the spread of COVID-19 is different from the situations considered in the research synthesised in this review. First, for the vast majority of the population, the required physical distancing leads to a much more pronounced lack of social connection than what they have experienced before. Second, physical distancing is externally imposed and not due to individual lifestyle decisions, lack of material means, poor social skills or other barriers to socialise. And third, physical distancing is 
requested from people in an overall context of uncertainty that comes with further stressors, health risks and often a reduced accessibility of healthcare.

It is important to note that physical distancing is a broad umbrella term that incorporates a wide range of potential measures, with highly divergent implications for social routines. It can include a full lock down and curfew, specific guidelines for meetings and gatherings of people, physical distancing in public and a recommended or mandatory wearing of face masks. The type, degree and duration of physical distancing measures have been variable across countries and will affect how isolated different groups in the population become.

One can only speculate as to whether and, if so, to what extent the increased lack of social connections resulting from physical distancing measures in the current situation will have an even greater impact on health outcomes than has been suggested in this review. Arguably, an even greater impact can be expected for certain risk groups such as socially disadvantaged groups (eg, individuals in need of mental or physical healthcare or individuals with low income) who often face even more economic adversity than before the pandemic. Further research is required to identify which populations are at particular risk to suffer health problems as a result of physical distancing and to explore whether the resulting lack of social connections may-at least to some extent and in some people-be compensated through positive effects of the pandemic, such as strengthened local communities and increased options for online social activities. ${ }^{47} 50$

\section{CONCLUSIONS}

In governmental decisions about future physical distancing measures, a potential negative impact of the resulting physical isolation on the health of the population needs to be considered. The existing literature suggests that social isolation and loneliness may affect both physical and mental health outcomes and include an excess mortality. However, the potential impact of physical distancing on social isolation and loneliness and ultimately on physical and mental health outcomes need to be thoroughly examined. In addition, the existing knowledge on the association between social connection and physical and mental health should be considered in clinical practice. Finally, more experimental research is needed to increase our understanding of the causal relationship between social connection and physical and psychological well-being.

\section{Twitter Thole Hilko Hoppen @HoppenDr}

Contributors NM had full access to all of the data in the study and takes responsibility for the integrity of the data and the accuracy of the data analysis. NM designed the search strategy with input from AK and TM. NM and AK carried out the literature searches and screening. NM, THH and TM carried out the data extraction. AK and TM assessed the quality of the included meta-analyses. NM and SP wrote the first draft of the manuscript and all authors contributed to and have approved the final manuscript.

Funding The authors have not declared a specific grant for this research from any funding agency in the public, commercial or not-for-profit sectors.

Competing interests None declared.

Patient and public involvement Patients and/or the public were not involved in the design, or conduct, or reporting, or dissemination plans of this research.

Patient consent for publication Not required.

Provenance and peer review Not commissioned; externally peer reviewed.

Data availability statement Data sharing not applicable as no datasets generated and/or analysed for this study.

Open access This is an open access article distributed in accordance with the Creative Commons Attribution Non Commercial (CC BY-NC 4.0) license, which permits others to distribute, remix, adapt, build upon this work non-commercially, and license their derivative works on different terms, provided the original work is properly cited, appropriate credit is given, any changes made indicated, and the use is non-commercial. See: http://creativecommons.org/licenses/by-nc/4.0/.

\section{ORCID iDs}

Nexhmedin Morina http://orcid.org/0000-0002-2331-9140

Thole Hilko Hoppen http://orcid.org/0000-0002-6050-8696

\section{REFERENCES}

1 Holmes EA, O'Connor RC, Perry VH, et al. Multidisciplinary research priorities for the COVID-19 pandemic: a call for action for mental health science. Lancet Psychiatry 2020;7:547-60.

2 Holt-Lunstad J. Why social relationships are important for physical health: a systems approach to understanding and modifying risk and protection. Annu Rev Psychol 2018;69:437-58.

3 Perissinotto CM, Covinsky KE, alone L. Living alone, socially isolated or Lonely-What are we measuring? J Gen Intern Med 2014;29:1429-31.

4 Holt-Lunstad J, Smith TB, Baker M, et al. Loneliness and social isolation as risk factors for mortality: a meta-analytic review. Perspect Psychol Sci 2015;10:227-37.

5 National Academies of Sciences, Engineering, and Medicine. Social isolation and loneliness in older adults: opportunities for the health care system. Washington, DC: National Academies Press, 2020.

6 Hawkley LC, Wroblewski K, Kaiser T, et al. Are U.S. older adults getting lonelier? age, period, and cohort differences. Psychol Aging 2019;34:1144-57.

7 DiJulio B, Hamel L, Muñana C. Loneliness and social isolation in the United States, the United Kingdom, and Japan: an international survey. The Economist \& Kaiser Family Foundation, 2018.

8 Anderson GO, Thayer CE. Loneliness and social connections: a national survey of adults 45 and older. Washington, DC: AARP Foundation, 2018.

9 Perissinotto CM, Stijacic Cenzer I, Covinsky KE. Loneliness in older persons: a predictor of functional decline and death. Arch Intern Med 2012;172:1078-84.

10 Cacioppo JT, Cacioppo S, Capitanio JP, et al. The neuroendocrinology of social isolation. Annu Rev Psychol 2015;66:733-67.

11 Seeman TE, Singer BH, Ryff CD, et al. Social relationships, gender, and allostatic load across two age cohorts. Psychosom Med 2002;64:395-406.

12 Hawkley LC, Capitanio JP, isolation Psocial. Perceived social isolation, evolutionary fitness and health outcomes: a lifespan approach. Philos Trans R Soc Lond B Biol Sci 2015;370:20140114.

13 Grant N, Hamer M, Steptoe A. Social isolation and stress-related cardiovascular, lipid, and cortisol responses. Ann Behav Med 2009;37:29-37.

14 Tricco AC, Langlois EV, Straus SE others. Rapid reviews to strengthen health policy and systems: a practical guide. Geneva: World Health Organization, 2017.

15 Moher D, Liberati A, Tetzlaff J. Preferred reporting items for systematic reviews and meta-analyses: the PRISMA statement. Ann Intern Med 2009;151:264-9.

16 Shea BJ, Reeves BC, Wells G, et al. AMSTAR 2: a critical appraisal tool for systematic reviews that include randomised or nonrandomised studies of healthcare interventions, or both. BMJ 2017;358:j4008. 
17 Steptoe A, Kivimäki M. Stress and cardiovascular disease: an update on current knowledge. Annu Rev Public Health 2013;34:337-54.

18 Valtorta NK, Kanaan M, Gilbody S, et al. Loneliness and social isolation as risk factors for coronary heart disease and stroke: systematic review and meta-analysis of longitudinal observational studies. Heart 2016;102:1009-16.

19 Rico-Uribe LA, Caballero FF, Martín-María N, et al. Association of loneliness with all-cause mortality: a meta-analysis. PLoS One 2018;13:e0190033.

20 Smith KJ, Gavey S, Rlddell NE, et al. The association between loneliness, social isolation and inflammation: a systematic review and meta-analysis. Neurosci Biobehav Rev 2020;112:519-41.

21 Kojima G, Taniguchi Y, Kitamura A, et al. Is living alone a risk factor of frailty? A systematic review and meta-analysis. Ageing Res Rev 2020;59:101048.

22 Maes M, Van den Noortgate W, Fustolo-Gunnink SF, et al. Loneliness in children and adolescents with chronic physical conditions: a metaanalysis. J Pediatr Psychol 2017;42:622-35.

23 Besora-Moreno M, Llauradó E, Tarro L, et al. Social and economic factors and malnutrition or the risk of malnutrition in the elderly: a systematic review and meta-analysis of observational studies. Nutrients 2020;12:737.

24 Jain $A$, van Hoek AJ, Boccia $D$, et al. Lower vaccine uptake amongst older individuals living alone: a systematic review and meta-analysis of social determinants of vaccine uptake. Vaccine 2017;35:2315-28.

25 Heidari Gorji MA, Fatahian A, Farsavian A. The impact of perceived and objective social isolation on hospital readmission in patients with heart failure: a systematic review and meta-analysis of observational studies. Gen Hosp Psychiatry 2019;60:27-36.

26 Xiu-Ying H, Qian C, Xiao-Dong P, et al. Living arrangements and risk for late life depression: a meta-analysis of published literature. Int $J$ Psychiatry Med 2012;43:19-34.

27 Penninkilampi R, Casey A-N, Singh MF, et al. The association between social engagement, loneliness, and risk of dementia: a systematic review and meta-analysis. JAD 2018;66:1619-33.

28 Evans IEM, Martyr A, Collins R, et al. Social isolation and cognitive function in later life: a systematic review and meta-analysis. $J$ Alzheimers Dis 2019;70:S119-44.

29 Kuiper JS, Zuidersma M, Oude Voshaar RC, et al. Social relationships and risk of dementia: a systematic review and meta-analysis of longitudinal cohort studies. Ageing Res Rev 2015;22:39-57.

30 Lara E, Martín-María N, De la Torre-Luque A, et al. Does loneliness contribute to mild cognitive impairment and dementia? A systematic review and meta-analysis of longitudinal studies. Ageing Res Rev 2019;52:7-16.

31 Maes M, Nelemans SA, Danneel S, et al. Loneliness and social anxiety across childhood and adolescence: multilevel meta-analyses of cross-sectional and longitudinal associations. Dev Psychol 2019;55:1548-65.

32 Choi HJ, Smith RA, Members SRA. Members, isolates, and liaisons: meta-analysis of adolescents' network positions and their smoking behavior. Subst Use Misuse 2013;48:612-22.
33 Mahon NE, Yarcheski A, Yarcheski TJ, et al. A meta-analytic study of predictors for loneliness during adolescence. Nurs Res 2006;55:308-15.

34 Chang $\mathrm{Q}$, Chan $\mathrm{CH}$, Yip PSF. A meta-analytic review on social relationships and suicidal ideation among older adults. Soc Sci Med 2017;191:65-76.

35 Erzen E, Çikrikci Özkan, Ö Çikrikci. The effect of loneliness on depression: a meta-analysis. Int J Soc Psychiatry 2018;64:427-35.

36 Michalska da Rocha B, Rhodes S, Vasilopoulou E, et al. Loneliness in psychosis: a Meta-analytical review. Schizophr Bull 2018;44:114-25.

37 Chau AKC, Zhu C, So SH-W. Loneliness and the psychosis continuum: a meta-analysis on positive psychotic experiences and a meta-analysis on negative psychotic experiences. Int Rev Psychiatry 2019;31:471-90.

38 Teo AR, Lerrigo R, Rogers MAM. The role of social isolation in social anxiety disorder: a systematic review and meta-analysis. J Anxiety Disord 2013;27:353-64.

39 Chatterjee A, Banerjee S, Stein C, et al. Risk factors for depression among civilians after the 9/11 world Trade center terrorist attacks: a systematic review and meta-analysis. PLoS Curr 2018;10:ecurrents. dis.6a00b40c8ace0a6a0017361d7577c50a.

40 Yuan M-Z, Fang Q, Liu G-W, et al. Risk factors for post-acute coronary syndrome depression: a meta-analysis of observational studies. J Cardiovasc Nurs 2019;34:60-70.

41 Ong AD, Uchino BN, Wethington E. Loneliness and health in older adults: a mini-review and synthesis. Gerontology 2016;62:443-9.

42 Howick J, Kelly P, Kelly M. Establishing a causal link between social relationships and health using the Bradford Hill guidelines. SSM Popul Health 2019;8:100402.

43 Szreter S, Woolcock M. Health by association? social capital, social theory, and the political economy of public health. Int J Epidemiol 2004;33:650-67.

44 Craddock E, vanDellen MR, Novak SA, et al. Influence in relationships: a meta-analysis on health-related social control. Basic Appl Soc Psych 2015;37:118-30.

45 Postmes T, Wichmann LJ, van Valkengoed AM, et al. Social identification and depression: a meta-analysis. Eur J Soc Psychol 2019;49:110-26.

46 Uchino BN, Bowen K, de GRK. Social support and physical health: Models, mechanisms, and opportunities. In: Principles and concepts of behavioral medicine. Springer, 2018: 341-72.

47 Siette J, Cassidy M, Priebe S. Effectiveness of befriending interventions: a systematic review and meta-analysis. BMJ Open 2017;7:e014304.

48 Fakoya OA, McCorry NK, Donnelly M. Loneliness and social isolation interventions for older adults: a scoping review of reviews. BMC Public Health 2020;20:1-14.

49 Czaja SJ, Boot WR, Charness N, et al. Improving social support for older adults through technology: findings from the prism randomized controlled trial. Gerontologist 2018;58:467-77.

50 Steffens NK, LaRue CJ, Haslam C, et al. Social identification-building interventions to improve health: a systematic review and metaanalysis. Health Psychol Rev 2021;15:85-112. 